\title{
Outcome Measurements in Studies on the Association Between Osteoporosis and Periodontal Disease
}

Johelle de Santana Passos, ${ }^{*}$ Isaac Suzart Gomes-Filho, ${ }^{\dagger}$ Maria Isabel Pereira Vianna, ${ }^{\dagger}$ Simone Seixas da Cruz, $§$ Maurício Lima Barreto, ${ }^{\ddagger}$ Tiago José Silva Oliveira, ${ }^{*}$ Leandro Dias Borges," and Fernanda Marques Monteirol

Background: The present study aims to compare different diagnostic criteria for periodontal disease that were used to study the association between periodontal disease and osteoporosis.

Methods: A database from a case-control study was used. The present study included sociodemographic, lifestyle, and health-condition data; panoramic radiographs; and clinical measurements on periodontal conditions from 139 postmenopausal women. Each participant was classified with regard to the diagnosis of periodontal disease using five different criteria for the outcome measurement (OM) found in the literature in studies on the association between osteoporosis and periodontal disease (OM1 through OM5). The frequency of periodontal disease and diagnostic values were calculated taking OM5 as the gold standard and the main association measurement for all criteria using odds ratios.

Results: The frequency of periodontal disease varied from $24.5 \%$ to $98.6 \%$ depending on the OM used. OM4 and OM3 presented the highest specificity ( $98.9 \%$ and $94.5 \%$, respectively), whereas OM2 and OM1 presented the highest sensitivity (100\% and $77.1 \%$, respectively). The association measurements with adjustments for age and smoking varied significantly according to the OM over a range from 1.87 to 3.75 , and there were also variations in the statistical significance of the associations found.

Conclusions: The results of this study emphasize that the frequency of periodontal disease may be influenced by different OMs and indicated variations outside of the association between osteoporosis and periodontal disease. Therefore, there is a need for prior selection of a precise measurement of periodontal disease in investigations on this topic. J Periodontol 2010;81:17731780 .

\section{KEY WORDS}

Diagnosis; epidemiology; osteoporosis; periodontitis; reproducibility of results.

\footnotetext{
* Collective Health, Department of Health, Feira de Santana State University, Feira de Santana, Bahia, Brazil.

$\dagger$ Periodontics, Department of Health, Feira de Santana State University.

\# Epidemiology, Federal University of Bahia, Salvador, Bahia, Brazil.

$\S$ Epidemiology, Federal University of San Francisco Valley, Petrolina, Pernambuco, Brazil.

I| Dentistry, Department of Health, Feira de Santana State University.
}

The search for evidence that might reveal the influence of osteoporosis on the development of periodontal disease has gone on since the middle of the 1960s in several parts of the world. ${ }^{1}$ This topic has attained prominence within the sphere of worldwide public health because of the social costs and impact caused by bone fractures and tooth losses, respectively, and because of its proximity to aging. ${ }^{2}$

Osteoporosis is a multifactorial chronic disease and an important social and clinical problem that disables or incapacitates. Data on its prevalence are scarce and discontinuous. Some surveys estimated that it occurs in $15 \%$ to $20 \%$ of Brazilian women $>50$ years of age. ${ }^{3,4}$ It is characterized by a reduction in bone mineral density to below the minimum level required to ensure sufficient mechanical support, with consequent manifestations of pain, deformity, or fracture. It is also characterized by degeneration of the bone microarchitecture, which thus increases the fragility and risk of fracture. ${ }^{5}$

It was estimated that one in four women during menopause and one in three women $>65$ years of age are affected by osteoporosis. ${ }^{6}$ Around 10 million Brazilians have osteoporosis; 2.4 million of them suffer some type of fracture every year, and 200,000 of these 2.4 million people will die because 
of factors that result directly from such fractures by the year $2050 .^{7}$

With regard to periodontal disease, it has been shown that its worldwide prevalence is $10 \%$ to $15 \%$, although it may reach $80 \%$ in certain regions. ${ }^{8}$ Periodontal disease is considered to be the greatest cause of tooth loss and edentulism among adults, and it is more frequently observed in groups of older age individuals than in younger groups. Plaque development is greater and gingivitis is more severe in older individuals. ${ }^{8}$ Postmenopausal women with osteoporosis and periodontitis are also susceptible to an excessive response to dental plaque, as shown by greater bleeding on probing (BOP), dentoalveolar bone loss, and diminished alveolar bone mineral density. ${ }^{9}$ The multifactorial nature of periodontal disease means that certain systemic conditions (with osteoporosis among them) act as additional predisposing factors. Moreover, it was suggested that the systemic factors responsible for osteoporosis may interact with local factors to increase the pattern of alveolar bone loss. ${ }^{10}$

The association between osteoporosis (i.e., exposure) and periodontal disease (i.e., outcome) thus arises as a problem for investigation with regard to planning public policies because of the inevitable growth in demands on the health care system that this association entails, along with the inherent social and individual costs. Some studies ${ }^{11-13}$ showed a lack of association between osteoporosis and periodontal disease, whereas other studies ${ }^{10,14-17}$ revealed a significant association. The relationship between these diseases, along with the interfaces with other interfering variables, remains uncertain. Hence, population-based studies are necessary to achieve a better definition of the factors involved in this association and to plan prevention and control measures for these two diseases that have a great impact on health.

The controversy that exists regarding this topic may come from questions related to the methods used in studies, for example, the type of study, sample size calculation, data-analysis procedure, and exposure and outcome measurements (OMs). Although defined criteria for classifying the bone mineral density condition of individuals exist in relation to osteoporosis, ${ }^{5}$ regarding periodontal disease, a diversity of diagnostic methods for defining the presence of periodontitis exists. However, for studies on associations between pairs of diseases, it is an essential condition that the exposure measurement and the $\mathrm{OM}$ need to be robust to avoid the inclusion of false-positive individuals in the sample, which would change the final association measurement.

Along these lines, the present study aims to contribute toward the field of knowledge relating to the association between osteoporosis and periodontal disease by comparing different OMs (i.e., criteria for diag- nosing periodontal disease) because osteoporosis might be an important factor in the progression of this oral infection.

\section{MATERIALS AND METHODS}

\section{Sample}

The present study used the database from a casecontrol investigation ${ }^{15}$ that estimated the association between osteoporosis and periodontal disease among 139 postmenopausal women. The estimated sample size for the present study was calculated using software ${ }^{\text {II }}$ with a $95 \%$ confidence interval and power of $80 \%$. There were two controls for each case, in conformity with the parameters established for the prevalence of osteoporosis: $17 \%$ among the controls and $39 \%$ among the cases. ${ }^{18}$

Postmenopausal women ( $\geq 50$ years of age) who at tended the Assistance and Research Center to Human Reproduction, Feira de Santana, BA, Brazil, for bone densitometry testing, were approached and invited to participate in the study. Those women who showed an interest in this invitation were referred to the Dental Clinic of Feira de Santana State University (UEFS) after making prior appointments for evaluation of their oral condition. All participants received further information about the study protocol and objectives at the dental clinic. If they agreed to participate in the study, they signed an informed consent statement that authorized their inclusion in the study. Dental treatment was also provided on the basis of need.

Among the 174 women who sought an evaluation of their oral condition between June and November 2006, 35 women were excluded from the sample for this study because they presented diabetes $(n=12)$; edentulism $(n=7)$; or fewer than four teeth $(n=5)$; reached menopause $<1$ year earlier $(n=10)$; or did not agree to participate $(n=1)$. Thus, the final sample consisted of 139 women who fulfilled the following eligibility criteria: were $\geq 50$ years of age; had a densitometry report issued $<6$ months earlier; had a postmenopausal status for $\geq 1$ year; had $\geq 4$ teeth; were absent of systemic disease that might interfere with the inflammatory response, immune system, or bone metabolism, such as diabetes or kidney failure; or had a presence of bone lesions (tumors or osteomyelitis) in the dental arches. Thus, the case group $(n=48)$ was composed of postmenopausal women with periodontal disease, whereas the control group $(n=91)$ was composed of postmenopausal women without periodontal disease, although the proportions achieved did not exactly match the planned ratio $(2: 1)$ because of occurrences of losses over the course of the data gathering.

II EPI-Info software (version 6.0), CDC, Atlanta, GA. 
The study was approved by the Ethics Committee of the Science Development Foundation of Bahia, Salvador, BA, Brazil (protocol 047/2005).

\section{Data Gathering Procedures}

The women who agreed to participate in the study answered a questionnaire to obtain data relating to sociodemographic, biologic, and lifestyle factors such as: age, skin color, income, education level, physical activity practice, smoking habit, alcohol consumption, age at menopause, medical history, medications used, and oral habits. The women underwent a clinical dental examination and were sent to have panoramic radiographs produced for complementary evaluation of their oral condition. The panoramic radiograph was produced for all participants by using standardized techniques and processing at the same radiology service in the UEFS.

The densitometric reports on the participants were obtained, and certain information was extracted from them: weight, height, bone mineral density (in grams per square centimeters), and t score. Weights and heights were used to calculate the body mass index. Any diagnoses of osteoporosis were then recorded.

All clinical measurements of the groups that were defined were obtained by a single examiner (JdSP) who, at the time of the examination, was unaware of the bone mineral density of the women under evaluation. The reproducibility and concordance of the clinical measurements were calculated by means of the within-examiner $\kappa$ index for probing depth (PD) (0.6017) and recession/hyperplasia (0.6863) and between-examiner $\kappa$ index for PD (0.6080) and recession/hyperplasia (0.6671).

PD procedures were performed and recorded at six sites for each tooth as described by Pihlstrom et al. ${ }^{19}$ These consisted of four proximal measurements (mesio-vestibular, mesio-lingual, disto-vestibular, and disto-lingual), one measurement in the mid-vestibular region, and one measurement in the mid-lingual region. Measurements were made, using a probe ${ }^{\#}$ that was graduated in millimeters, of the distance from the gingival margin to the most apical extent of penetration of the probe. In addition, measurements of gingival recession/hyperplasia and clinical attachment loss (AL) were obtained at the mentioned sites using the same probe graduated in millimeters that was used to determine PD.

Gingival recession was defined as present when the gingival margin was located apically to the cementoenamel junction, and in such cases, the distance in millimeters from the gingival margin to the cementoenamel junction was taken to be positive. Hyperplasia was defined as present when the gingival margin was located coronally to the cemento-enamel junction, and in such cases, the distance in millimeters from the gingival margin to the cemento-enamel junction was taken to be negative. The clinical attachment measurement was taken to be the sum of the PD and the measurements of gingival recession or hyperplasia. In addition, BOP was determined as the rate observed at the six sites mentioned (i.e., whether bleeding occurred within 10 seconds after removing the probe from the pocket or sulcus).

\section{Classification of Periodontal Disease}

Each participant was classified according to the diagnosis of periodontitis. The frequency of periodontal disease was obtained using five diagnostic criteria presented in studies ${ }^{13,15,20-22}$ that investigated the association between osteoporosis and periodontal disease. Thus, five OMs were defined, as described in Table 1.

At the end of the data-gathering process, with all information and clinical-radiograph data at hand, the case and control groups were determined.

\section{Data Analysis Procedures}

For the data analyses, the cases of periodontal disease were distributed according to each OM. First, OM5 was taken to be the gold standard because it was the most comprehensive measurement. OM5 was observed using panoramic radiographs and a ruler graduated in millimeters with an expansion of $25 \%$. Thus, OM5 was used to make comparisons with the other measurements using the diagnostic values of sensitivity, specificity, positive-predictive value, and negative value.

Through stratified analyses, the existence of potential confounders and effect modifiers was investigated in relation to the following covariables: age, age at menarche, age at menopause, type of menopause, duration of menopause, skin color, family income, marital status, conjugal situation, parity, education level, physical activity practice, alcohol consumption, smoking habit, osteoporosis treatment, family history of osteoporosis, number of people living in the home, arterial hypertension, heart disease, coffee consumption, milk consumption, last visit to a dentist, use of dental floss, periodic consultations with a dentist, tooth loss because of caries, periodontal disease, and trauma.

Multivariate analyses were also performed to evaluate the statistical significance by means of unconditional logistic regression using backward procedures with a $95 \%$ confidence interval. Possible effect modifiers were evaluated by means of the maximum-likelihood ratio test. To analyze confounding factors, the association measurements estimated from the complete and partial models were compared. Covariables that produced proportional differences in the

\# Hu-Friedy PQW6, Hu-Friedy, Chicago, IL. 
Table I.

\section{Diagnostic Criteria for Periodontal Disease}

\begin{tabular}{|c|c|c|}
\hline OM & Criteria & Periodontal Protocol \\
\hline $1^{20}$ & $\geq 1$ site with $P D \geq 4 \mathrm{~mm}$ & $\begin{array}{l}\text { Six sites on the index teeth: \#2, \#3, \#8, } \\
\quad \#|4, \#| 5, \# \mid 8, \# 19, \# 24, \# 30, \\
\text { and \#31 }\end{array}$ \\
\hline $2^{13}$ & $\geq$ I vestibular site with clinical $A L \geq 3 \mathrm{~mm}$ & $\begin{array}{l}\text { Three vestibular sites per tooth (mesio- } \\
\text { vestibular, disto-vestibular, and mid- } \\
\text { vestibular) }\end{array}$ \\
\hline $3^{21}$ & $\begin{array}{l}\geq 2 \text { proximal sites with } P D \geq 5 \mathrm{~mm} \text { and } \\
\text { clinical } A L \geq 6 \mathrm{~mm} \text { at the same sites }\end{array}$ & $\begin{array}{l}\text { Four proximal sites per tooth (mesio- } \\
\text { vestibular, mesio-lingual, disto-vestibular, } \\
\text { and disto-lingual) }\end{array}$ \\
\hline $4^{22}$ & $\begin{array}{l}\geq 4 \text { teeth with } \geq 1 \text { site with } P D \\
\quad \geq 4 \mathrm{~mm} \text {, clinical } A L \geq 3 \mathrm{~mm} \text {, and } \mathrm{BOP}\end{array}$ & Six sites per tooth \\
\hline $5^{15}$ & $\begin{array}{l}\text { OM4 + periodontal bone reabsorption } \\
\geq 3 \mathrm{~mm} \text { apically in relation to the } \\
\text { cemento-enamel junction in } \geq 4 \text { teeth } \\
\text { at } \geq 1 \text { site }\end{array}$ & Six sites per tooth \\
\hline
\end{tabular}

all of the individuals, whereas OM3 was capable of identifying only around 25. Conversely, OM2 showed the lowest specificity and OM4 showed the highest specificity. Thus, there was a decrease in specificity from OM4 to OM2, which is the same as saying that there was a decrease in the capacity of the diagnostic criteria to identify individuals without periodontitis, i.e., of 100 individuals detected as not presenting periodontitis using the gold standard criteria (OM5), OM4 was able to identify $\approx 99$ individuals as negative. Likewise, there was also a decrease in the positive predictive value for the same measurements, i.e., a decrease in the likelihood of correctly identifying individuals with periodontitis using the OMs

estimated odds ratios $>20 \%$ were considered to be confounders. Data analyses were performed using a statistical software program. * *

\section{RESULTS}

Table 2 shows selected general characteristics taken from the whole database to demonstrate the nature of the study sample. Among other features, Table 2 shows that just over one-half of the study group consisted of women aged $\leq 58$ years and that menopause occurred at ages $\leq 48$ years in more than one-half. Likewise, it was also seen that women who were not living with a partner, had $\leq 5$ children, and lived with $\leq 3$ people in the same home. Furthermore, it was observed that the majority of women had a family income $\geq 1$ minimum monthly salary, had a middle or secondary school education level $\leq 4$ years, were non-smokers, were not alcohol consumers, did not practice physical exercise, had a body mass index $\leq 30 \mathrm{~kg} / \mathrm{cm}^{2}$, and did not consult a dentist regularly.

The mean number of teeth present in the sample was $\approx 14$. Regarding the periodontal condition, it was observed that the prevalence of periodontal disease ranged from $24.5 \%$ to $98.6 \%$ according to the diagnostic measurement used. This is presented in Table 3, which shows that the highest frequency was obtained with $\mathrm{OM} 2$, whereas the lowest frequency was obtained with OM3. By taking OM5 to be the gold standard, OM2 was the method with the greatest sensitivity, followed by OM4, OM1, and OM3, respectively. In other words, for every 100 individuals who were detected as having periodontitis by using the OM5 standard, OM2 was able to positively identify tested in relation to the gold standard, respectively, and a consequent increase in false positive results. With regard to the likelihood that an individual was disease free, with a negative result from the diagnostic criterion (negative-predictive value), it was observed that, in a general manner, the proportion of false-negative results ranged from $0 \%$ for OM2 to $18 \%$ for OM3.

Table 4 presents the association measurements between osteoporosis and periodontal disease, with adjustment for age and smoking, and the distribution of periodontal disease between case and control subjects. The strength of the association varied significantly depending on $\mathrm{OM}$. This variation had a range from 1.87 to 3.75 , and there were also statistically significant variations in the association found according to the measurement used. OM2 did not generate an association measurement value because periodontal disease was practically common to all of the women, i.e., it did not discriminate regarding the disease (98.6\% of the sample had periodontal disease, and $1.4 \%$ did not.). Thus, when diagnostic criteria were stricter, there were fewer occurrences of the disease in both groups, and consequently, the association measurement was more precise, as represented by confidence intervals.

Age and smoking habit were considered to be confounders from a theoretical point of view because they are classically recognized as factors associated with osteoporosis and periodontal disease. Adjustment for these factors resulted in a slight increase in the association measurements. Possible effect modifiers

\footnotetext{
* * Stata (version 8.0), StataCorp, College Station, TX.
} 
Table 2.

\section{Sociodemographic and Lifestyle Characteristics ( $N=139)$}

\begin{tabular}{|c|c|c|}
\hline Characteristics & $\mathrm{n}$ & $\%$ \\
\hline \multicolumn{3}{|l|}{ Age (years) } \\
\hline$\leq 58$ & 75 & 54.0 \\
\hline$>58$ & 64 & 46.0 \\
\hline \multicolumn{3}{|l|}{ Age at menopause } \\
\hline$>48$ years & 58 & 41.7 \\
\hline$\leq 48$ years & 81 & 58.3 \\
\hline \multicolumn{3}{|l|}{$\mathrm{BMI}\left(\mathrm{kg} / \mathrm{cm}^{2}\right)$} \\
\hline$\leq 30$ & 114 & 82.0 \\
\hline$>30$ & 25 & 18.0 \\
\hline \multicolumn{3}{|l|}{ Skin color } \\
\hline White/Asian & 34 & 24.5 \\
\hline African American/biracial & 105 & 75.5 \\
\hline \multicolumn{3}{|l|}{ Marital status } \\
\hline With partner & 67 & 48.2 \\
\hline Without partner & 72 & 51.8 \\
\hline \multicolumn{3}{|l|}{ Family income } \\
\hline$\geq 1$ minimum salary & 128 & 92.1 \\
\hline$<$ minimum salary & 11 & 7.9 \\
\hline \multicolumn{3}{|l|}{ Education level } \\
\hline$>4$ years & 39 & 28.1 \\
\hline$\leq 4$ years & 100 & 71.9 \\
\hline \multicolumn{3}{|l|}{ Number of children } \\
\hline$\leq 5$ children & 75 & 54.0 \\
\hline$>5$ children & 64 & 46.0 \\
\hline \multicolumn{3}{|c|}{$\begin{array}{l}\text { Number of people living in the } \\
\text { home }\end{array}$} \\
\hline$\leq 3$ people & 78 & 56.1 \\
\hline$>3$ people & 61 & 43.9 \\
\hline \multicolumn{3}{|l|}{ Smoking } \\
\hline No & 129 & 92.8 \\
\hline Yes & 10 & 7.2 \\
\hline \multicolumn{3}{|l|}{ Alcohol use } \\
\hline No & 106 & 76.3 \\
\hline Yes & 33 & 23.7 \\
\hline \multicolumn{3}{|l|}{ Physical activity practice } \\
\hline No & 88 & 63.3 \\
\hline Yes & 51 & 36.7 \\
\hline \multicolumn{3}{|c|}{ Regular consultation with a dentist } \\
\hline No & 121 & 87.0 \\
\hline Yes & 18 & 13.0 \\
\hline
\end{tabular}

$\mathrm{BMI}=$ body mass index.

were evaluated, and education level was identified as an interaction factor for OM4 and OM5. After stratification, it was observed that, for both measurements, there was an association between osteoporosis and periodontal disease and for a level of education of $\leq 4$ years of study, which presented statistical significance.

\section{DISCUSSION}

The findings from the present study indicate that the strength of the association between osteoporosis and periodontal disease varied according to the type of OM used, and the statistical significance also varied. The different measurements influenced the magnitude of the association and may also have influenced the frequency of periodontal disease.

At the outset, it is important to highlight the choice of the clinical and radiographic criteria put forward by Gomes-Filho et al. ${ }^{15}$ as the gold standard. These diagnostic criteria for periodontal disease use an association of periodontal bone reabsorption $(\geq 3 \mathrm{~mm}$ ) with three clinical descriptors for the disease: PD ( $\geq 4 \mathrm{~mm})$, clinical AL ( $\geq 3 \mathrm{~mm})$, and BOP (present). Furthermore, using these criteria, periodontitis was defined as present when $\geq 4$ teeth presented all these descriptors in $\geq 1$ site. Thus, this definition for the diagnosis of periodontal disease is rigorous, which is an essential precondition for studies on associations between periodontitis and systemic conditions or abnormalities.

Moreover, these proposed criteria were used in investigating associations in which periodontitis was considered to be the exposure factor ${ }^{22}$ or the OM. ${ }^{15}$ In these studies, ${ }^{15,22}$ the diagnostic value of specificity presented by these criteria was good, which is an essential precondition for reducing the rate of falsepositive diagnoses among participants in groups with periodontal disease.

Among the five sets of diagnostic criteria used for defining periodontal disease, which were extracted from studies on this topic, ${ }^{13,15,20-22}$ OM2 (i.e., $\geq 1$ vestibular site with clinical $A L \geq 3 \mathrm{~mm}$ ) was present in practically all of the women evaluated. Thus, OM2 did not discriminate between case and control groups (with and without periodontal disease, respectively), and for this reason, no association measurement was obtained. In other words, the diagnostic criteria for periodontal disease used by Famili et al. ${ }^{13}$ are overly sensitive and include many false positives with a diagnosis of periodontitis. This may have been the reason why these authors ${ }^{13}$ were led to conclude in their study that there was little evidence for an association between periodontal disease and abnormalities of bone mineral density.

In evaluating OM4 (i.e., a minimum of four teeth with $\geq 1$ site presenting $\mathrm{PD} \geq 4 \mathrm{~mm}$ with clinical $\mathrm{AL}$ $\geq 3 \mathrm{~mm}$ at the same sites and $\mathrm{BOP}^{22}$ ), it was observed that the final association measurement only presented statistical significance for the stratum of women whose education level was $\geq 4$ years. This finding should be viewed with caution because the 
Table 3.

Distribution of Periodontal Disease, Diagnostic Values, and Confidence Intervals (CIs) for OM1, OM2, OM3, and OM4 Compared With OM5 ( $\mathrm{N}=139)$

\begin{tabular}{ccccccc}
\hline OM & $\begin{array}{c}\text { Case of } \\
\text { PD }(\mathrm{N})\end{array}$ & $\begin{array}{c}\text { Frequency } \\
\text { of PD }(\%)\end{array}$ & $\begin{array}{c}\text { Sensitivity } \\
(95 \% \mathrm{Cl})(\%)\end{array}$ & $\begin{array}{c}\text { Specificity } \\
(95 \% \mathrm{Cl})(\%)\end{array}$ & $\begin{array}{c}\text { Positive Predictive } \\
\text { Value }(95 \% \mathrm{Cl})(\%)\end{array}$ & $\begin{array}{c}\text { Negative Predictive } \\
\text { Value }(95 \% \mathrm{Cl})(\%)\end{array}$ \\
\hline 1 & 64 & 46.0 & $77.1(62.3$ to 87.5$)$ & $70.3(59.7$ to 79.2$)$ & $57.8(44.8$ to 69.8$)$ & $85.3(74.8$ to 92.1$)$ \\
2 & 137 & 98.6 & $100(90.8$ to 100$)$ & $2.2(0.4$ to 8.5$)$ & $35(27.2$ to 43.7$)$ & $100(19.8$ to 100) \\
3 & 34 & 24.5 & $60.4(45.3$ to 73.9$)$ & $94.5(87.1$ to 98.0$)$ & $85.3(68.2$ to 94.5$)$ & $81.9(72.9$ to 88.5$)$ \\
4 & 36 & 25.9 & $79.2(57.9$ to 84.3$)$ & $98.9(93.2$ to 99.9$)$ & $97.2(83.8$ to 99.9$)$ & $87.4(79.0$ to 92.8$)$ \\
5 & 48 & 34.5 & - & - & - & - \\
\hline
\end{tabular}

Table 4.

Distribution of Periodontal Disease Between Case and Control Groups, Unadjusted and Adjusted Odds Ratios (ORs), and Confidence Intervals (CIs) for the Association Between Osteoporosis and Periodontal Disease (OM1 through OM5) ( $N=139)$

\begin{tabular}{|c|c|c|c|c|c|c|c|c|c|c|}
\hline \multirow[b]{2}{*}{ OM } & \multirow[b]{2}{*}{$\begin{array}{l}\text { Cases } \\
(\mathrm{n}[\%])\end{array}$} & \multirow[b]{2}{*}{$\begin{array}{l}\text { Controls } \\
\text { (n [\%]) }\end{array}$} & \multirow[b]{2}{*}{$\begin{array}{c}\text { Unadjusted } \\
\text { OR }\end{array}$} & \multirow[b]{2}{*}{$95 \% \mathrm{Cl}$} & \multirow[b]{2}{*}{$\begin{array}{c}\text { Adjusted } \\
\text { Overall } O R^{*}\end{array}$} & \multirow[b]{2}{*}{$95 \% \mathrm{Cl}$} & \multicolumn{2}{|c|}{$\leq 4$ Years of Education } & \multicolumn{2}{|c|}{$>4$ Years of Education } \\
\hline & & & & & & & $\begin{array}{c}\text { Adjusted } \\
\mathrm{OR}^{*}\end{array}$ & $95 \% \mathrm{Cl}$ & $\begin{array}{c}\text { Adjusted } \\
\text { OR* }\end{array}$ & $95 \% \mathrm{Cl}$ \\
\hline I & $64(46.0)$ & $75(54.0)$ & 2.08 & 0.96 to 4.51 & 2.27 & 1.03 to 5.05 & - & - & - & - \\
\hline 2 & 137 (98.6) & $2(1.4)$ & - & - & - & - & - & - & - & - \\
\hline 3 & $34(24.5)$ & 105 (75.5) & 3.75 & 1.22 to 11.49 & 3.90 & 1.26 to 12.06 & - & - & - & - \\
\hline 4 & $36(25.9)$ & $103(74.1)$ & 1.87 & 0.74 to 4.71 & 2.02 & 0.79 to 5.19 & 4.71 & 1.21 to 18.37 & 0.55 & 0.11 to 2.64 \\
\hline 5 & $48(65.5)$ & 91 (34.5) & 2.58 & 1.08 to 6.19 & 2.71 & 1.12 to 6.56 & 7.52 & 1.99 to 28.35 & 0.50 & 0.11 to 2.05 \\
\hline
\end{tabular}

* Adjusted for smoking habit and age.

statistical analyses included subjects with fewer teeth, and the effects from socioeconomic status, such as education level, regularity of consultations with a dentist, or unknown factors that were not investigated in this study, may have had interactions with the small numbers of teeth. Furthermore, because the sample size was smaller than that calculated initially for the study, it is likely that the power of the study was a factor that influenced the final finding for this type of measurement. After controlling for the influence of other factors that could have modified the strength of the association for this stratum of education level, OM4 presented statistical significance. In the diagnostic evaluation, it was noted that OM4 presented very good specificity compared to the gold standard.

However, in studies in which periodontal disease is the outcome, local factors may influence the diagnosis of periodontitis, such as the presence of calculi or excessive numbers of tooth restorations, among others. In such situations, complementary radiographs may assist in defining the effect. In the present study, panoramic radiographs that presented enlarged images were used. However, to neutralize the magnification factor of $25 \%$ in these radiographs, a ruler graduated in millimeters was incorporated in these images as a precaution. Furthermore, the radiographic criteria consisted of a minimum of four teeth with $\geq 1$ site that presented bone loss $\geq 3 \mathrm{~mm}$ as measured from the cemento-enamel junction. Thus, the diagnosis was not based on only one or two sites with AL because such a situation could be related to local factors such as excessive restoration material or some other factor leading to retention of bacterial biofilm. OM5 corroborated the findings from a study ${ }^{16}$ that used the same criteria for OMs.

On the other hand, OM3 (i.e., $\geq 2$ sites with PD $\geq 5$ $\mathrm{mm}$ and clinical $\mathrm{AL} \geq 6 \mathrm{~mm}$ at the same sites) was the set of criteria that presented the greatest severity of periodontal disease. This resulted in a greater strength of association because it is easier 
to diagnose periodontal disease when the effects of periodontal disease are more severe. On the contrary, many women who presented mild to moderate severity of periodontal disease did not form part of the case group, which thus gave rise to possible distortions in the association measurements. The present results diverge from the results found in a study $^{21}$ that used these criteria for diagnosing periodontal disease because the authors of the study concluded that women who received estrogen supplementation presented diminished gingival inflammation and AL.

The adjusted association measurement using the OM1 criteria (i.e., PD $\geq 4 \mathrm{~mm}$ measured at six sites on the index teeth \#2, \#3, \#8, \#14, \#15, \#18, \#19, $\# 24$, \#30, and \#31) presented statistical significance. However, these diagnostic criteria came from a partial examination of the mouth by using only six of 10 index teeth, and they do not represent the individual's real condition. These criteria were designed to evaluate the need for periodontal treatment in populationbased studies. Their use is justified for evaluating periodontal care in large populations (i.e., in epidemiologic studies that serve as the basis for planning oral healthcare actions), and this partial examination has good indications for such purposes. The findings from this measurement corroborate those obtained by Inagaki et al. ${ }^{20}$

With regard to the standardization of the exposure measurement, it is important to emphasize that all densitometry tests were performed at the same osteoporosis diagnostic service, with the same equipment and under technical quality control, with the aim of obtaining greater uniformity of results. Furthermore, in the analyses, the covariables of age and smoking habit were kept in the modeling as confounding factors, independent of the results from empirical analysis. This is indicative of the care taken in interpreting the findings, along with the use of education level as an interaction factor for OM4 and OM5.

For the findings presented here to be better interpreted, certain limitations on the data deserve to be discussed. Among these limitations were the small numbers of teeth presented by the individuals examined. In addition, the sample was small and, consequently, had a low power to detect the real effect of osteoporosis on periodontal conditions. For inclusion in this study, participants had to present a minimum of four teeth, which minimized the number of false-positive cases when further combined with the three clinical periodontal descriptors of PD, clinical attachment level, and gingival bleeding. Moreover, the Brazilian population is mostly characterized by significant tooth loss, which is the historical result from caries. Hence, setting a cutoff point for subject inclusion of a minimum of 20 teeth, for example, would have restricted the sample and implied difficulties of eligibility and, furthermore, the non-representativeness of the population.

Criteria of greater robustness for studies on the association between osteoporosis and periodontal disease should envisage the complete examination of all teeth, at all six sites, with simultaneous use of the greatest number of periodontal descriptors possible (clinical attachment level, PD, and BOP) to achieve the best possible characterization of the presence of periodontitis through periodontal support loss and the presence of inflammation. Such criteria should incorporate a minimum number of compromised teeth and at least a moderate level of severity. Once the present work is an epidemiologic study, and it would be difficult to use imaging resources for diagnoses, it is further suggested that, whenever possible, radiographs should be used to confirm the presence of bone reabsorption. In this manner, it would be easier to avoid the possibility of including individuals with a false-positive diagnosis of periodontal disease.

Lastly, from a methodologic point of view, the choice of diagnostic criteria partly reflects the internal validity of the study because the disease classification may introduce measurement errors and, thus, cause a bias in the association measurement, compromise the quality of measurements, and make it difficult to accumulate consensual knowledge around hypotheses that are put forward at the interface between medicine and dentistry.

\section{CONCLUSIONS}

According to the results from this investigation, epidemiologic measurements may be influenced by the outcome classification used (i.e., the diagnostic criteria for periodontal disease). In other words, it was observed that different findings can be obtained from association studies when different definitions for the factors involved are used. Thus, investigators need to carry out prior evaluations and tests on the diagnostic criteria that are to be used in their studies so that these criteria are sufficiently robust to avoid fragility in developing the method.

\section{ACKNOWLEDGMENTS}

The authors acknowledge Dr. Marcelo Esteve, the manager responsible for the institution, which the postmenopausal women in this study attended and all the staff of the Assistance and Research Center to Human Reproduction, Feira de Santana, BA, Brazil. We also acknowledge the Research Support Foundation of the State of Bahia, the National Council for Scientific and Technological Development, and UEFS for financial support for the research. The authors report no conflicts of interest related to this study. 


\section{REFERENCES}

1. Groen JJ, Menczel J, Shapiro S. Chronic destructive periodontal disease in patients with presenile osteoporosis. J Periodontol 1968;39:19-23.

2. Reddy MS. Osteoporosis and periodontitis: Discussion, conclusions, and recommendations. Ann Periodontol 2001;6:214-217.

3. National Consensus Proposal. Osteoporosis 1995 Basic diagnosis and therapeutic elements. São Paulo Med J 1995;113:7-18.

4. Costa-Paiva L, Horovitz AP, Santos Ade O, FonsechiCarvasan GA, Pinto-Neto AM. Prevalence of osteoporosis in post menopausal women and association with clinical and reproductive factors. Rev Bras Ginecol Obstet 2003;25:507-512.

5. World Health Organization. Assessment of Fracture Risk and Its Application to Screening for Postmenopausal Osteoporosis. Report of a WHO Study Group. Technical Reports Series, No. 843. Geneva: WHO; 1994:1-129.

6. Pallos D, Ceschin A, Victor GA, Bulhões RC, Quirino MRS. Menopause: A risk factor for periodontal disease? (in Portuguese) Rev Bras Ginecol Obstet 2006;28:292297.

7. Brazilian Society of Osteoporosis. Bulletin (in Portuguese). Official Publication of the Brazilian Society of Osteoporosis 2004;15:1-7.

8. Kinane DF, Peterson M, Stathopoulou G. Environmental and other modifying factors of the periodontal disease. Periodontol 2000 2006;40:107-119.

9. Payne JB, Reinhardt RA, Nummikoski PV, Patil KD. Longitudinal alveolar bone loss in postmenopausal osteoporotic/osteopenic women. Osteoporos Int 1999; 10:34-40.

10. Tezal M, Wactawski-Wende J, Grossi SG, Ho AW, Dunford R, Genco RJ. The relationship between bone mineral density and periodontitis in postmenopausal women. J Periodontol 2000;71:1492-1498.

11. Ronderos M, Jacobs DR, Himes JH, Pihlstrom BL. Associations of periodontal disease with femoral bone mineral density and estrogen replacement therapy: Cross-sectional evaluation of US adults from NHANES III. J Clin Periodontol 2000;27:778-786.

12. Pilgram TK, Hildebolt CF, Dotson M, et al. Relationships between clinical attachment level and spine and hip bone mineral density: Data from healthy postmenopausal women. J Periodontol 2002;73:298-301.
13. Famili P, Cauley J, Suzuki JB, Weyant R. Longitudinal study of periodontal disease and edentulism with rates of bone loss in older women. J Periodontol 2005;76: 11-15.

14. Wactawski-Wende J, Hausmann E, Hovey K, Trevisan M, Grossi S, Genco RJ. The association between osteoporosis and alveolar crestal height in postmenopausal women. J Periodontol 2005;76:2116-2124.

15. Gomes-Filho IS, Passos JS, Cruz SS, et al. The association between postmenopausal osteoporosis and periodontal disease. J Periodontol 2007;78:1731 1740.

16. Brennan-Calanan RM, Genco RJ, Wilding GE, Hovey KM, Trevisan M, Wactawski-Wende J. Osteoporosis and oral infection: Independent risk factors for oral bone loss. J Dent Res 2008;78:323-327.

17. Haas AN, Rösing CK, Oppermann RV, Albandar JM, Susin C. Association among menopause, hormone replacement therapy, and periodontal attachment loss in southern Brazilian women. J Periodontol 2009;80: 1380-1387.

18. Inagaki K, Kurosu Y, Yoshinari N, Noguchi T, Krall EA, Garcia RI. Efficacy of periodontal disease and tooth loss to screen for low bone mineral density in Japanese women. Calcif Tissue Int 2005;77:9-14.

19. Pihlstrom BL, Ortiz-Campos C, McHugh RB. A randomized four-year study of periodontal therapy. J Periodontol 1981;52:227-242.

20. Inagaki K, Kurosu Y, Kamiya T, et al. Low metacarpal bone density, tooth loss, and periodontal disease in Japanese women. J Dent Res 2001;80:1818-1822.

21. Reinhardt RA, Payne JB, Maze CA, Patil KD, Gallagher SJ, Mattson JS. Influence of estrogen and osteopenia/ osteoporosis on clinical periodontitis in postmenopausal women. J Periodontol 1999;70:823-828.

22. Gomes-Filho IS, Cruz SS, Rezende EJ, et al. Exposure measurement in the association between periodontal disease and prematurity/low birth weight. J Clin Periodontol 2007;34:957-963.

Correspondence: Dr. Johelle de Santana Passos, Avenida Getúlio Vargas, 379, Centro, Feira de Santana 44.025010, BA, Brazil. Fax: 55-75-3623-0661; e-mail: johpassos@ gmail.com.

Submitted March 10, 2010; accepted for publication June 22, 2010 